\title{
Wide-band detector for micro-microampere low-energy electron currents
}

\author{
by T. E. EVERHART, Ph.D., * and R. F. M. THORNLEY, B.A., Department of Engineering, \\ University of Cambridge
}

[Paper received 25 January, 1960]

\begin{abstract}
Electrons with a mean energy of a few electron volts emerging from a source a few millimetres in diameter are accelerated on to a positively biased plastic scintillator. The light generated in the scintillator is guided by a Perspex light-pipe to a photomultiplier. If the scintillator bias is greater than $10 \mathrm{kV}$, no noise is introduced by the scintillator-light-pipe-photomultiplier system. If the original electron signal is modulated, the maximum modulation frequency transmitted by the system is greater than $10 \mathrm{Mc} / \mathrm{s}$. The minimum detectable current is limited by the photomultiplier dark current, and may be less than $10^{-15} A$ with a suitably chosen tube.
\end{abstract}

\section{Introduction}

1 HE properties of the detector described in this paper were largely determined by the instrument for which it was designed, the scanning electron microscope. In this instrument, a fine electron probe is scanned in a rectangular raster across the surface to be examined. Secondary electrons leaving the surface are collected, amplified and used to control the brightness of a cathode-ray tube scanned in synchronism with the probe. Details of the instrument and the contrast mechanism have been published by McMullan, (1) Smith and Oatley, (2) and Everhart, Wells and Oatley. (3) For the full capabilities of the instrument to be realized, relatively noise-free amplification of the modulated secondary electron current is required. As this current ranges from $10^{-13}$ to $10^{-10} \mathrm{~A}$, and contains frequency components up to $200 \mathrm{kc} / \mathrm{s}$, conventional thermionic amplifiers cannot comply with the low-noise and bandwidth requirements. Early workers used secondary emission electron multipliers, but these were not very satisfactory, electrically or mechanically.

\section{General description}

The new detector (shown in Fig. 1) consists of a cylindrical brass shield which is closed at the end facing the specimen by a grid of copper gauze (etched in nitric acid to increase the grid porosity) and is biased positively in order to attract the low-energy electrons. Once through the grid, the electrons are accelerated toward the hemisphere of plastic scintillator, the surface of which is covered with a $700 \AA$ layer of aluminium (maintained at 7 to $12 \mathrm{kV}$ positive). The intense electrostatic field, shaped by the focusing electrode,

* Now at Department of Electrical Engineering, University of California, U.S.A. causes most electrons to strike the hemisphere near its apex. The light generated in the scintillator is guided by a Perspex light pipe to a commercial photomultiplier tube which then converts the light back to an electron signal and provides most of the required amplification.

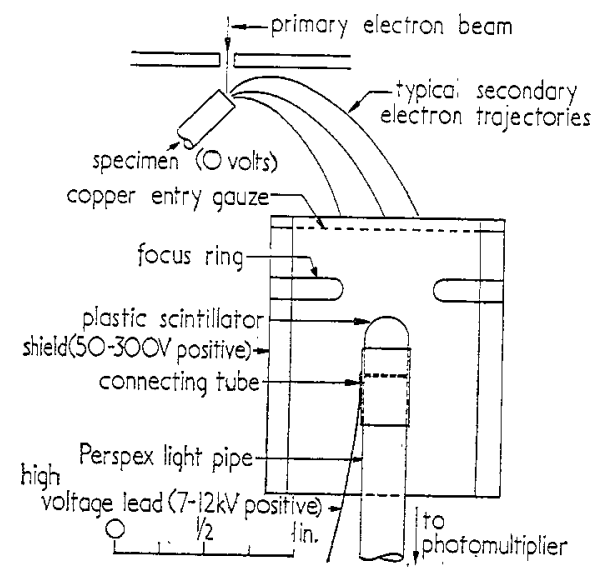

Fig. 1. Diagram showing collector geometry

\section{Theoretical performance}

For such a system to be reasonably noise-free, each electron entering the collector must liberate, on the average, at least one electron from the photomultiplier cathode. The number of photoelectrons produced by one zero-energy secondary electron entering the collector is given by:

$$
n=\left\{\left(E_{s}-E_{a}\right) C_{p} C_{0} C_{e} f\left(\nu_{p}\right)\right\}: E_{p}
$$

where $E_{s}=$ energy of the electron striking the scintillator surface $(\mathrm{eV})$,

$E_{a}=$ energy lost by the electron in passing through the aluminium film $(\mathrm{eV})$,

$C_{p}=$ energy conversion efficiency into photons of average energy $E_{p}$,

$C_{0}=$ efficiency of the optical system,

$C_{e}=$ conversion efficiency of the photocathode,

$f(v)=$ spectral response of the photocathode to photons of energy $E_{p}(\mathrm{eV})$.

$E_{a}$ can be calculated from the Thompson-Whiddington law:

$$
E_{a}=\sqrt{ }\left(E_{s}^{2}-a x d\right)
$$

where $x$ is the film thickness in $\mathrm{cm}, a$ is $4 \times 10^{11}(\mathrm{eV})^{2} \mathrm{~cm}^{2} / \mathrm{g}$, and $d$ is the density in $\mathrm{g} / \mathrm{cm}^{3}$. 
In the early work on the collector, the manufacturer's figure for $C_{p}$ was used and $C_{0}$ was assumed to be unity. This gave $n$ equal to $2 \cdot 2$ for $E_{s}=7 \mathrm{kV}$, indicating that under these conditions no noise was introduced. It was found in practice that noise was being introduced and a detailed investigation of $C_{p}$ and $C_{0}$ was carried out.

\section{Scintillator efficiency}

Several workers ${ }^{(4-6)}$ have reported that the conversion efficiency of scintillators falls as the incident particle energy is reduced below $50 \mathrm{keV}$, but the losses quoted vary considerably. It was therefore necessary to measure $C_{p}$ at the energy levels in question.

Two makes of plastic scintillator were investigatedPamelon, made by Isotopes Development Ltd.; and Naton 11, made by Nash and Thompson Ltd. No significant difference in performance was detected.

A $10 \mathrm{kV}$ electron beam carrying about $10^{-12} \mathrm{~A}$ was arranged to strike the scintillator surface, the potential of which could be varied between $\div 10$ and $-10 \mathrm{kV}$. It was found that the response varied approximately linearly with incident energy for energies above a threshold value which varied between 2 and $10 \mathrm{kV}$. Typical curves are shown in Fig. 2. Further investigation showed that the method used

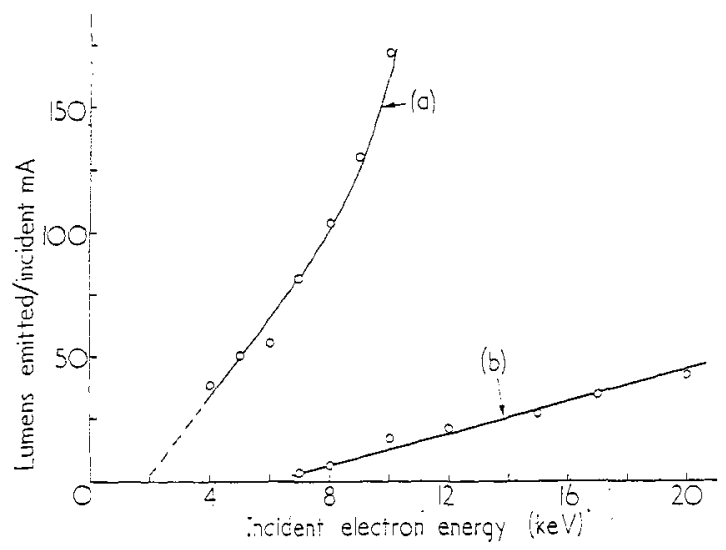

Fig. 2. Scintillator performance

Curve (a), wet machined, French chalk polished; curve (b), dry machined, polished with metal polish

to prepare the scintillator surface was of critical importance in determining the performance for low incident energies. Scintillators machined under water-cooled conditions and polished with French chalk in water showed threshold energies of 2 to $2.5 \mathrm{keV}$. This energy is that required to penetrate the $700 \AA$ aluminium film used to make the scintillator surface conducting. Dry machining and/or polishing with proprietary brands of metal polish raised the threshold into the 5 to $10 \mathrm{keV}$ range. Any slight overheating during the metallizing had a similar deleterious effect on the performance.

Measurements with a calibrated photomultiplier showed that the conversion efficiency of a well prepared scintillator was $0.02 \pm 0.005$ for $7 \mathrm{keV}$ electrons. This figure agrees, within the limits of experimental error, with the manufacturer's value.

The scintillator did not show any saturation effects until the input current density reached about $1 \mathrm{~A} / \mathrm{cm}^{2}$. Under

VOL. 37, JULY 1960 normal operating conditions, the input current density is less than $1 \mu \mathrm{A} / \mathrm{cm}^{2}$ so saturation is not a limiting factor in the performance of the collector.

\section{Optical system efficiency}

The optical efficiency of the scintillator-light-pipe system is estimated by assuming that all electrons entering the collector shield (see Fig. 1) strike the scintillator near the apex of the hemisphere. The critical angle $\theta_{c}$ of the scintillator material (measured from a surface normal to the ray path) is $40 \cdot 5^{\circ}$. If no light is absorbed by the scintillator, and no loss occurs at surface reflexions, then by using a simple geometrical argument, over $90 \%$ of the generated light is found to enter the light-pipe. Further, all these light rays entering the light-pipe subtend an angle to the surface normal greater than the critical angle of the light-pipe material, Perspex. Thus in the absence of absorption in the light-pipe, all light entering the pipe should reach the photomultiplier. If it is assumed that a $10 \%$ loss of intensity occurs at each surface reflexion in the scintillator, about $80 \%$ of the generated light enters the light-pipe.

The transmission efficiency of the light-pipes used was measured with a special neon light source having an intensity distribution similar to that expected from a scintillator hemisphere. The light-pipes were made from $\frac{3}{8}$ in. diameter Perspex rod cut in lengths varying from 9 to $11 \mathrm{~cm}$. The ends were polished. Bends of $45^{\circ}$ and $90^{\circ}$ with a radius of $2.5 \mathrm{~cm}$ were made on some light-pipes by gentiy warming the rod in a low bunsen flame and then bending to the desired shape. It was found that in no case did the transmission exceed $65 \%$, and if extreme care was not taken with the surface finish, the transmission fell below $40 \%$. The bends did not increase the loss by more than $10 \%$ in any case. This loss, which is rather surprising in view of the high transparency of Perspex, is believed to be mainly caused by reflexion losses on entering and leaving the pipe. A drop of vacuum oil placed in a joint between two pipes increased the transmission by about $5 \%$ provided the oil did not spread along the ferrule supporting the joint. If this occurred, the loss was greater than for a dry joint.

\section{Measured performance}

Putting $C_{0}=0.4, C_{D}=0.02$ and $n=1$ in the transfer equation gives $E_{s}=8 \mathrm{keV}$. This analysis depends on the accuracy of the photomultiplier calibration which was not better than $20 \%$. An independent check on the result was made by observing the signal/noise ratio of the photomultiplier output for a constant input current, with the scintillator voltage varied to give values of $E_{s}$ between 0 and $20 \mathrm{keV}$. This gave the curve shown in Fig. 3, indicating

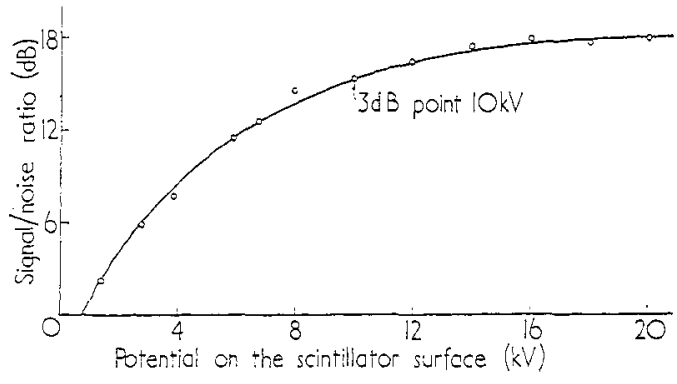

Fig. 3. Signal/noise ratio variation with scintillator potential 
that the noise is effectively constant for scintillator potentials greater than $10 \mathrm{kV}$. (The $18 \mathrm{~dB}$ signal-to-noise ratio is determined by the shot noise in the primary electron beam plus the noise introduced in the secondary emission process.)

\section{Conclusions}

A wide-band detector for micro-microampere secondary electron currents has been constructed and tested. Noisefree current gains ranging from $10^{5}$ to $10^{8}$ have been observed with a bandwidth exceeding $200 \mathrm{kc} / \mathrm{s}$. The minimum signal which can be detected is set by the dark current of the photomultiplier used. For the tube used in this investigation, an E.M.I. type 6094B, this dark current is less than $10^{-15} \mathrm{~A}$, and it could be reduced further by cooling the photocathode.

The maximum bandwidth is set by the decay time of the scintillator or the transit time spread of the photomultiplier, or both. The decay time for Pamelon is stated to be less than $10^{-8} \mathrm{~s}$, and the transit time spread for the photomultiplier used is about $10^{-8} \mathrm{~s}$. Thus the bandwidth of the present system is estimated to be greater than $10 \mathrm{Mc} / \mathrm{s}$.

As used in the scanning electron microscope, this detector offers important advantages. The mechanical flexibility is much better than that of the old secondary electron detector. A movement of the detector input requires only a new piece of light-pipe bent to the proper shape with the new detector, while previously an entire new brass bottom plate for the specimen chamber had to be constructed.

Previously, the input of the electron multiplier was necessarily near ground potential, requiring the output to be approximately $6 \mathrm{kV}$ positive. This in turn required that the initial stages of valve amplification float at $6 \mathrm{kV}$ positive. With the new detector, the output of the photomultiplier can be at ground potential, making a direct-coupled videoamplifier possible.

\section{Acknowledgements}

We wish to express our thanks to our supervisor, Mr. C. W. Oatley, for much useful advice and encouragement.

One of us (T. E. E.) was supported by a Marshall Scholarship, while the other (R. F. M. T.) was in receipt of a D.S.I.R. Research Studentship.

\section{References}

(1) McMullan, D. Proc. Instn Elect. Engrs, 100, Part II, p. 245 (1953).

(2) Smith, K. C. A., and OAtley, C. W. Brit. J. Appl. Phys., 6, p. 391 (1955).

(3) Everhart, T. E., Wells, O. C., and Oatley, C. W. J. Elect. \& Control, 7, p. 97 (1959).

(4) TAYLOR, C. J., JeNTSChKE, W. K., REMLEY, M. E., EBY, F. S., and Kruger, P. G. Phys. Rev., 84, p. 1034 (1951).

(5) BiRks, J. B., and King, J. W. Phys. Rev., 86, p. 568 (1952).

(6) BeLCHER, E. H. Brit. J. Radiol., 30, p. 103 (1957).

\section{LABORATORY AND WORKSHOP NOTES}

\section{Modified cylindrical cell for the microelectrophoresis of suspended particles}

by E. J. Ambrose, M.A., and J. Ryder, B.Sc., Chester Beatty Research Institute, Institute of Cancer Research: Royal Cancer Hospital, London, S.W.3

\section{[Note received 6 January, 1960]}

Satisfactory chambers for the measurement of the electrical mobility of very small particles have been known for some time. (1) An apparatus suitable for the measurement of suspended bacteria with a rectangular chamber $(0.5 \times 25 \mathrm{~mm}$ cross-section) has been described by Loveday and James. ${ }^{(2)}$ In these cases the particles remain continuously in suspension and they can always be observed at the stationary level, where the true electrophoretic mobility can be measured. The stationary level lies at a distance of 0.21 times the total depth of chamber from the chamber wall and parallel to the wali. Larger particles sediment under gravity and their mobility can only be measured accurately if they are allowed to fall freely, in the plane of the stationary level, while the electric field is applied at right angles to this direction. The rectangular chamber must therefore be arranged vertically so that the particles can fall through the whole depth of the chamber. (3) This arrangement is reasonably satisfactory but is most sensitive to small thermal changes owing to the vertical depth $(25 \mathrm{~mm})^{(4)}$ within which convection currents can arise. The location of the large particles (up to $20 \mu$ in diameter) at the stationary level in the small total depth $(500 \mu)$ of the chamber also presents difficulties.
Bangham, Flemans, Heard and Seaman ${ }^{(5)}$ described an apparatus using a cylindrical chamber which has several advantages. The height of the chamber is only equal to the diameter of the tube $(2.33 \mathrm{~mm})$ and convection currents leading to drift are therefore not troublesome. The depth of the chamber $(2330 \mu)$ is relatively much larger in relation to the diameter of the particles $(20 \mu)$. The apparatus is satisfactory for moderately large particles, such as red blood cells, but larger particles sediment extremely rapidly in the small depth of the tube and it is difficult to take more than ten readings after filling the chamber, even when working at great speed. To enable more readings to be taken, and with greater accuracy, a method of resuspending the cells in the chamber has been devised.

A small piece of Mumetal (approximately $3 \mathrm{~mm}$ long) sealed into thin-walled capillary tubing (Fig. 1) is placed in

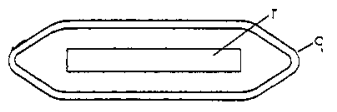

Fig. 1. Enlarged view of stirrer $r$, Mumetal; $q$, thin glass

the electrophoresis chamber. It is drawn along through the chamber by a magnet (Fig. 2). Its passage stirs up the suspension and resuspends the cells without disturbing the thermal equilibrium. The magnet is moved along a metal 